\title{
Author Correction: Cryo-electron tomography reveals that dynactin recruits a team of dyneins for processive motility
}

Danielle A. Grotjahn, Saikat Chowdhury (D, Yiru Xu, Richard J. McKenney, Trina A. Schroer and Gabriel C. Lander (D)

Nat. Struct. Mol. Biol. https://doi.org/10.1038/s41594-018-0027-7; published online 07 February 2018

In the version of this article initially published online, an incorrect accession code, EMD-5NW4, was introduced on page 1 of the article $\mathrm{PDF}$, in section 'BICD2N mediates the association of two dynein dimers with a single dynactin'. This has been corrected to PDB 5NW4.

The error has been corrected in the PDF and HTML versions of this article.

\section{Author Correction: Structural basis of AAUAAA polyadenylation signal recognition by the human CPSF complex}

\author{
Marcello Clerici (D), Marco Faini, Lena M. Muckenfuss, Ruedi Aebersold (D) and Martin Jinek (DD
}

Nat. Struct. Mol. Biol. https://doi.org/10.1038/s41594-017-0020-6; published online 22 January 2018.

In the version of this article initially published online, an incorrect accession code PDB 6FN9 was introduced in Methods, in the 'Model building' section, line 2. This has been corrected to PDB 6F9N. The error has been corrected in the PDF and HTML versions of this article.

Published online: 14 March 2018

https://doi.org/10.1038/s41594-018-0044-6

\section{Author Correction: Global delay in nascent strand DNA methylation \\ Jocelyn Charlton, Timothy L. Downing (1), Zachary D. Smith, Hongcang Gu, Kendell Clement, Ramona Pop, Veronika Akopian, Sven Klages, David P. Santos, Alexander M. Tsankov, Bernd Timmermann, Michael J. Ziller, Evangelos Kiskinis, Andreas Gnirke and Alexander Meissner (D)}

Correction to: Nature Structural \& Molecular Biology https://doi.org/10.1038/s41594-018-0046-4, published online 12 March 2018.

Following online publication of this article, the Gene Expression Omnibus records corresponding to accession codes GSM2406773, MN-d6, and GSM2406772, MN-d14, listed in the data availability statement were deleted. The data are now available under accession codes GSM3039355, WGBS_hESC_WT_D6_R4 (MN day 6), and GSM3039351, WGBS_hESC_WT_D14_R4 (MN day 14), and the data availability statement has been updated with the new accession codes in the HTML and PDF versions of the article.

Published online: 26 March 2018

https://doi.org/10.1038/s41594-018-0055-3 